\title{
Determinación de la seroprevalencia de Salmonella spp. en granjas porcinas del departamento del Tolima
}

\author{
Determining Salmonella spp. seroprevalence on pig-farms in the \\ Tolima department
}

\section{Determinação da seroprevalencia de Salmonella spp. em granjas porcinas do departamento do Tolima}

\author{
lang S Rondón-Barragán ${ }^{1 *}$, Gustavo A Rodríguez², Gira A Marín M $^{1 *}$ \\ MVZ, MSc, Universidad del Tolima, Facultad de Medicina Veterinaria y Zootecnia - Departamento de Sanidad Animal \\ MVZ, Instituto Colombiano Agropecuario (ICA). \\ Grupo de Investigación en Inmunobiología y Patogénesis - GIP. Laboratorio de Diagnóstico Veterinario \\ Email: isrondon@ut.edu.co
}

Recibido: Julio 5 de 2012. Aceptado: Diciembre 18 de 2013.

\begin{abstract}
Resumen
La salmonelosis es una enfermedad bacteriana de distribución mundial, importante en salud pública, siendo una de las patologías que se presenta frecuentemente en humanos y animales, causante de grandes pérdidas económicas en el sector porcícola. El presente reporte busca visualizar la situación actual de la seroprevalencia de la Salmonella spp. En el Departamento del Tolima. Para tal fin se analizaron un total de 9 granjas porcinas distribuidas en el Departamento. Recolectando de manera aleatoria muestras individuales $(n=420)$ de sangre de animales en diferentes fases productivas (iniciación, levante, ceba, adultos reproductores), sin distinción de sexo; para ser evaluadas por medio de pruebas serológicas (ELISA indirecto) comercialmente disponibles. Los animales muestreados no presentaron signos clínicos compatibles con salmonelosis. La seroprevalencia de Salmonella hallada fue de $36.09 \%$.
\end{abstract}

Palabras clave: cerdos, Salmonella, seroprevalencia, ETAs.

\begin{abstract}
Salmonella is a bacterial disease having worldwide distribution; it has an important effect on public health, being one of the diseases which is frequently found in humans and animals, causing large-scale economic loses in the pig-farming sector. The present report was aimed at raising awareness of the current situation regarding Salmonella spp. seroprevalence in the Tolima department. Nine pig-farms distributed throughout the department were thus analysed. Individual blood samples $(n=420)$ were randomly taken from animals in different production phases (initiation, weaning, fattening, reproductive adults), regardless of gender, to be serologically evaluated using commercially available tests (indirect ELISA). The sampled animals did not have clinical signs which were compatibles with salmonella. Salmonella seroprevalence was $36.09 \%$.
\end{abstract}

Key words: pigs, Salmonella, seroprevalence, ETA. 


\section{Resumo}

A salmonela é uma doença bacteriana do mundo de distribuição, importante em saúde pública, sendo uma das patologias que muitas vezes ocorre em humanos e animais, causando grandes prejuízos económicos no sector da carne de porco. Este relatório pretende exibir o status atual da prevalência de Salmonella spp. no departamento de Tolima. Um total de 9 criações de porcos em todo o departamento foram analisadas para o efeito. Recolha de forma aleatória, amostras individuais $(n=420)$ sangue de animais em diferentes fases produtivas (iniciação, elevação, engorda, adultos reprodutores), sem distinção de sexo; a ser avaliada por meio de testes sorológicos (ELISA indireta) comercialmente disponíveis. Amostra de animais não apresentaram sinais clínicos compatíveis com salmonelose. A prevalência encontrada que Salmonella foi 36.09\%.

Palavras-chave: suínos, Salmonella, soroprevalência, ETAs.

\section{Introducción}

La salmonelosis es una de las enfermedades zoonóticas más comunes y de mayor importancia económica a nivel mundial, es producida por enterobacterias del género Salmonella que afectan igualmente un amplio rango de hospederos animales: insectos, reptiles, aves y mamíferos (Hollinger, 2000; Barrow y Mathner, 2013). Anualmente se ha estimado la presentación de 93.8 millones de casos de gastroenteritis en humanos por infección con Salmonella y 155.000 muertes a nivel mundial (Majowicz et al., 2010). Para el año 2009 se reportaron en la Unión Europea 108.614 casos de salmonelosis humana siendo la segunda enfermedad zoonótica más frecuente, antecedida por la campilobacteriosis con 198.552 casos reportados (European Food Safety Authority-EFSA, 2010)

La Salmonella es trasmitida por medio la vía fecal-oral puesto que la mayor fuente de contaminación son las heces de animales (Hollinger, 2000; Quinn et al., 2001). La infección se genera a partir la ingestión de alimento o agua contaminada con dicho patógeno, o a través del contacto directo o indirecto con animales infectados (Hendriksen et al., 2011; Hoelzer et al., 2011).

Esta enfermedad bacteriana causa un amplio número de manifestaciones clínicas: salmonelosis tifoidal: fiebre entérica y salmonelosis no tifoidal: gastroenteritis, bacteremia, infección focal extra intestinal y estado portador crónico (Langridge et al., 2008). La gastroenteritis o enterocolitis agudas es la forma de presentación más común en humanos; los síntomas aparecen generalmente 6 a 48 horas posteriores al consumo de alimentos contaminados e incluyen diarrea, cefalea, dolor abdominal, náuseas, vómito, fiebre y deshidratación, afectando principalmente niños menores de 5 años y adultos mayores de 65 años de edad (Cummings et al., 2010).

Existen solo dos especies de Salmonella: S. bongori y enterica (caracterizadas por hibridización de ADN) de las cuales han sido reconocidos más de 2600 serovares de acuerdo a sus reacciones serológicas al lipopolisacárido somático $(\mathrm{O})$, antígeno flagelar $(\mathrm{H})$ y antígeno capsular (Vi) (Grimont y Weill, 2007; Guibourdenche et al., 2010). Para S. enterica existen 6 subespecies: enterica, salamae, arizonae, diarizonae, houtenae, indica; siendo la subespecie enterica serovar Typhimurium, la más común en animales domésticos y el ser humano (Quinn et al., 2001; Hur et al., 2012), seguida de los serovares $S$. Enteritidis, $S$. Newport y $S$. Heidelberg, estos ampliamente patogénicos en humanos (Hur et al., 2012).

En animales la salmonelosis puede ocurrir de forma epizoótica, enzoótica o esporádica. Las especies de Salmonella más frecuentemente aisladas de animales enfermos incluyen S. typhimurium, S. enteritidis, $S$. dublin, S. cholerasuis y S. typhosa. Todos los serovares de Salmonella son considerados potencialmente patógenos en la mayoría de las especies animales. Sin embargo, la patogenicidad de algunos serovares parece estar limitada a un estrecho rango de hospederas animales y son considerados adaptados a especies, tales como $S$. dublin en el ganado (Hollinger, 2000).

En la especie canina, Rincón-Reyes y Figueroa (2008) reportaron una prevalencia serológica de SalmoneIla enteritidis del $41,7 \%$ en Tunja; describiendo a la presentación de problemas entéricos como uno de los principales factores de riesgo relacionados con la seropositividad. En pollos se ha determinado una prevalencia en broilers del $20 \%$ y en reproductores del $20 \%$ (Rusul et al., 1996). Sallam et al., (2014) determinaron una prevalencia general de Salmonella del $24.4 \%$ en carne de res fresca, carne molida de res y hamburguesas de ternera. Adicionalmente, en estudios realizados en el caribe colombiano a partir de alimentos obtenidos de ventas comidas callejeras y plazas de mercado se encontró una prevalencia de Salmonella del 7,4\%.; del total de muestras de chorizo, el 12,6\% fueron positivas para Salmonella spp., 10,5\% de arepa de huevo, 
$9,3 \%$ de carne de res, $7,9 \%$ de queso, $5,2 \%$ de carne de cerdo, y $1,6 \%$ de pollo (Durango et al., 2004).

Además de los huevos contaminados y las aves, el cerdo y su productos son reconocidos como una de las mayores fuentes de salmonelosis humana (Grebeyes et al., 2008; Cardinale et al., 2010). Y su presencia en la cadena de cárnicos porcina ha sido reconocida, así como identificados diversos factores de riesgo asociados a ella (Cardinale et al., 2010; Henao \& Ramírez, 2010). En la Unión Europea, a diferencia de los países de Latinoamérica, existe un programa de vigilancia activa de la salmonelosis porcina que ha llevado a crear el índice de Salmonella, así como a establecer planes de contingencia frente a este (Alban et al., 2012).

En el país son tan escasos como necesarios los estudios epidemiológicos relacionados con enfermedades bacterianas como la salmonelosis. Es por este motivo que el presente trabajo tiene como objetivo determinar la seroprevalencia de Salmonella spp. En el Departamento del Tolima, en diferentes etapas del ciclo de producción porcina. Este trabajo hace parte de un proyecto marco denominado Evaluación epidemiológica de la presencia de Salmonella spp. y determinación de factores de riesgo asociados a su presentación en granjas del Departamento del Tolima, apoyado por el Ministerio de Agricultura y Desarrollo Rural (MADR) y la Asociación Colombiana de Porcicultores (ACP).

\section{Materiales y métodos}

\section{Ubicación}

El estudio se realizó en granjas porcícolas ubicadas en el Departamento del Tolima, localizado en el centro oeste de Colombia, entre las cordilleras Central y Occidental.

\section{Animales}

Para el estudio se muestrearon animales $(n=420)$ de las diferentes fases de producción en las grajas porcinas que incluyeron: reproductores, cerdas gestantes, cerdas lactantes, cerdas descanso, lactantes, precebo, levante, ceba, cerdas de reemplazo y prejaulón partos. Los animales fueron seleccionados al azar.

\section{Muestreo}

La selección de las granjas se realizó a través del método no probabilístico, buscando la representatividad en el Departamento del Tolima, de tal manera que se muestrearon las regiones Norte, Sur, Oriente, Occidente y Centro. Se muestrearon granjas registra- das en la base de datos de la Asociación Colombiana de Porcicultores y que correspondieran a sistemas tecnificados (i.e. aquellas que llevaran registros, separación por edades, asesoría técnica, entre otros). Los animales fueron seleccionados completamente al azar y de forma proporcional al número de animales en la granja, distribuidos en las diferentes fases de producción.

El tamaño de la muestra se calculó a través de la fórmula descrita por Thrusfield (2007), contando con un nivel de confianza del $95 \%$, un $50 \%$ de seroprevalencia esperada en cerdos y una precisión del 10\%.

$$
n=\frac{z^{2} \times p \times q}{d^{2}}
$$

Dónde:

$\mathbf{z}^{2}=$ coeficiente del nivel de confianza prefijado $\left(1.96^{2}\right.$ ya que el nivel de confianza es de $95 \%$ )

$p=$ proporción esperada (en este estudio $50 \%=0.50$ )

$q=1-p$

d $=$ precisión (en este estudio un 5\%)

Para un total de 384 animales. En el presente estudio se muestrearon 420 animales.

Se tomaron muestras sanguíneas de los animales previamente inmovilizados de acuerdo al protocolo establecido por el Laboratorio de Diagnóstico Veterinario de la Facultad de Veterinaria y Zootecnia de la Universidad del Tolima, evitando el maltrato de los mismos. La sangre se extrajo de la vena yugular o de la oreja, según se mostró conveniente con cada animal en particular. La sangre se depositó en tubos vacutainer sin anticoagulante y se conservaron en refrigeración durante su transporte al laboratorio para ser centrifugadas. Posteriormente, el suero fue separado y congelado a $-30^{\circ} \mathrm{C}$ para luego ser utilizado en una prueba de ELISA indirecta para Salmonella mediante un kit comercial (HerdChek* Swine Salmonella IDEXX ${ }^{\circledR}$ ).

\section{Prueba de ELISA}

Para la determinación de anticuerpos séricos contra Salmonella spp. se realizó la prueba de ELISA indirecta utilizando un kit comercial HerdChek* Swine Salmonella (Especificidad: 0.994, Sensibilidad: 0.57; IDEXX ${ }^{\circledR}$, Alemania) en placas de 96 pozos. Esta prueba se realizó en el Laboratorio de Diagnóstico Veterinario de la Universidad del Tolima, mediante el uso de un lector de placas ELx800 (Biotek ${ }^{\circledR}$ Instruments, Inc.) empleando un filtro de $650 \mathrm{~nm}$ y el punto de corte de densidad óptica (OD) se ajustó a lo descrito en otros trabajos (Alban et al., 2002). 


\section{Análisis estadístico}

El análisis estadístico comprendió una evaluación descriptiva de los datos, la determinación de la seroprevalencia, así como un prueba de Chi cuadrado $\left(x^{2}\right)$ para establecer la correlación existente entre el ciclo de los animales muestreados y la seropositividad a Salmonella spp. Los ciclos fueron categorizados de la siguiente manera: ciclo $1=$ reproductores; $2=$ gestación; $3=$ cerdas lactantes; $4=$ cerdas descanso; $5=$ =lactantes; $6=$ precebo; $7=$ levante; $8=$ ceba; $9=$ cerdas reemplazo; $11=$ prejaulón partos. Los datos fueron analizados estadísticamente mediante el uso del software estadístico SAS versión 8.0 para Windows (SAS, USA) y graficados a través de Stat graphics Centurion XV versión 15.2.05 para Windows ( ${ }^{\odot}$ StatPoint, Inc, 2007).

\section{Resultados}

En el presente estudio ninguno de los animales muestreados evidenció signos clínicos compatibles con salmonelosis, ni con alguna otra enfermedad entérica o sistémica. De la misma manera, no presentaron episodios febriles al momento del muestreo y no se reportaron antecedentes sanitarios asociados a los mismos. Dado que las granjas no presentaban un tamaño uniforme, y como se especificó en la metodología, se tomaron muestras de diferentes etapas del sistema de producción. El número de muestras fue dependiente del tamaño de la producción, puesto que las explotaciones de mayor tamaño requerían un número mayor de muestras representativas de la misma. Así la distribución de estas muestras fue desbalanceada proporcional a cada grupo.
La etapa del ciclo de producción con mayor número de animales muestreados fue la del ciclo 11, seguido del ciclo 7 y 6 . El ciclo de menor participación con animales fue el ciclo 1 y 4 con un porcentaje de 2,25\% para cada uno. Este desbalance se presenta ya que no en todas las explotaciones la distribución de animales era homogénea.

La seroprevalencia obtenida en granjas porcinas del Departamento del Tolima fue del $36.09 \%$, con una mayor seroprevalencia en los individuos de las etapa de gestación y de ceba (Figura 1).

\section{Discusión}

En animales domésticos, incluyendo el cerdo, la Salmonella puede causar a espectro de expresión de enfermedad, desde una infección sub-aparente, productividad alterada o enfermedad clínica que puede variar de leve a fatal en severidad (Tunes \& Blas, 2007). De igual forma, se han descrito distintos patrones de distribución, virulencia, patogénesis y resistencia, adscritos a serovares específicos o incluso a clasificaciones subserotípicas de Salmonella, basados en fagotipos, perfiles de plásmidos, perfiles de susceptibilidad antimicrobiana u otros métodos de diferenciación (Hollinger, 2000).

El aislamiento del microorganismo en animales puede resultar dificultoso cuando se presenta en bajo número debido a la competencia por sobrecrecimiento de las Enterobacteriaceas (Hollinger, 2000). Por lo cual el análisis serológico surge como alternativa para el diagnóstico de la presencia o prevalencia de la bacteria en

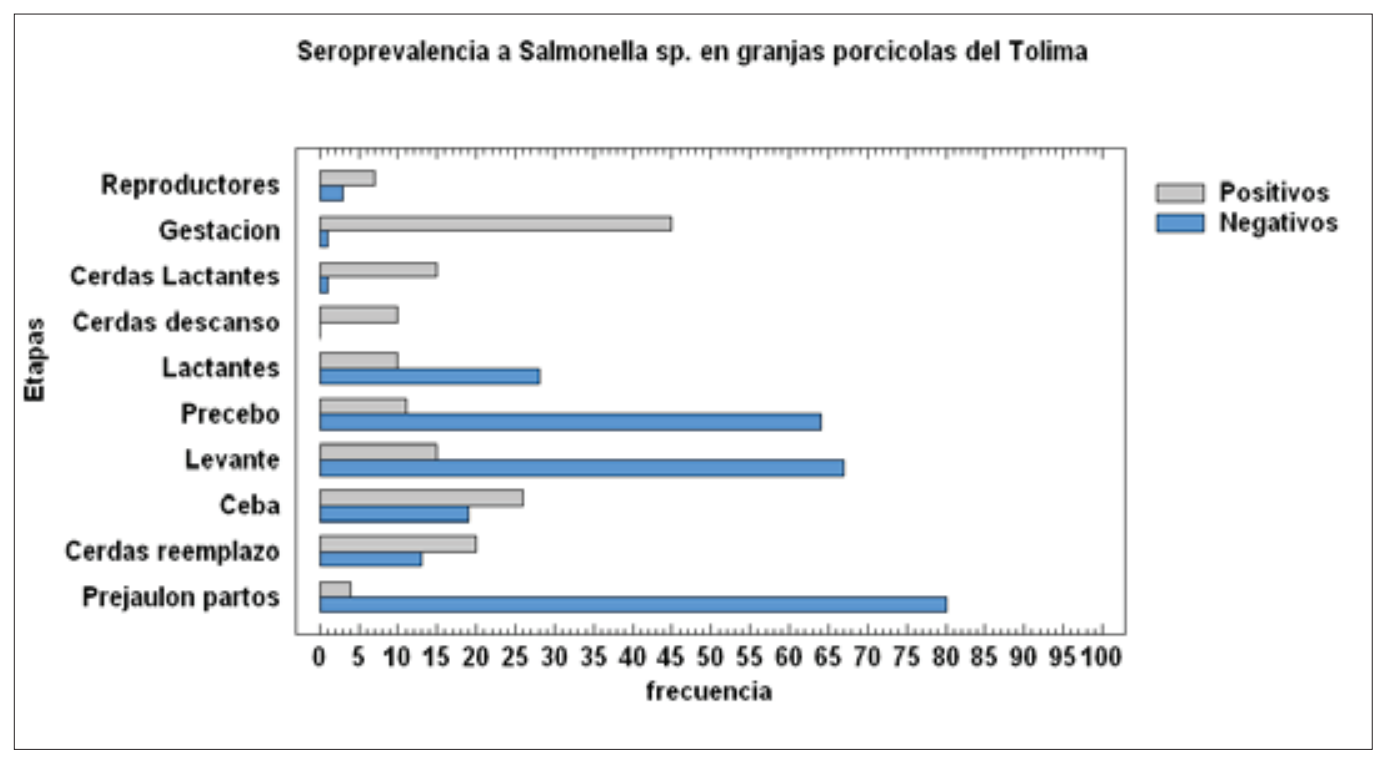

Figura 1. Distribución de la población muestreal por ciclos y su respectiva serología a Salmonella spp. Nótese la mayor positividad en los animales de gestación, ceba, levante y cerdas de reemplazo. 
una población. No obstante, la determinación serológica para la presencia de infección posee sus limitaciones. El periodo de latencia entre la exposición y la presencia de anticuerpos, interferencia por inmunoglobulinas transferidas pasivamente, la incapacidad para identificar el serovar infectante y la reactividad cruzada con anticuerpos debido a la inmunización. Además la determinación de la tasa de infección, usando técnicas de aislamiento o serología, puede resultar difícil para evaluar poblaciones afectadas (Hollinger, 2000). Sørensen et al., (2004) evaluaron la relación entre la serología del mix-ELISA y el aislamiento de SalmoneIla, encontrando unas correlaciones moderadas entre la positividad del Mix-ELISA con el aislamiento en el contenido cecal, superficie de las carcasas y faringe; no así para el aislamiento de nódulos linfoides cecales. Así ellos sugieren que los diagnósticos serológicos deben ser utilizados para la vigilancia pre-comercial de los sistemas de producción. Así mismo Wong et al., (2003) encontraron una correlación moderada entre los animales seropositivos y los aislamientos fecales de Salmonella. No obstante, Funk et al., (2005), describen que ni el cultivo a partir de muestras fecales ni la detección de anticuerpos séricos representan una prueba diagnóstica perfecta, dado que no existió una correlación entre los hallazgos de cada una de la pruebas y la presencia de la enfermedad. Los investigadores infieren que la prueba diagnóstica dependerá, no de sus características, mas sí de su propósito de prueba per se (evaluación de poblaciones, evaluación de la eficacia de las intervenciones, rastreo de brotes de origen alimentario, monitoreo o control de procesos). De la misma manera, Nowak et al., (2007) compararon la técnica de ELISA con el PCR para diagnóstico de Salmonella. Así describen que las disminuciones del punto de corte en el ELISA de 40 a 20 pueden facilitar la detección de granjas problemas en el sentido de seropositividad en títulos, pero disminuye la correlación de animales seropositivos con el número de animales que estén verdaderamente enfermos (esto contrastado con sus hallazgos en PCR en las heces). Sallam et al.,(2014) reportaron una mayor prevalencia de muestras positivas de Salmonella detectadas por PCR en comparación con el método convencional de cultivo bacteriológico, indicándose así una mayor sensibilidad del PCR.

Por otro lado, los cerdos infectados pueden excretar gran número de Salmonella en las heces. Incluso se considera, que la mayoría de las veces la infección con Salmonella es subclínica y la excreción es intermitente, con bajos niveles de bacteria lo cual puede guiar a resultados falsos negativos (Kranker et al., 2003). Consecuentemente, en los estadios tempranos de infección, los animales pueden ser positivos a las pruebas bacteriológicas y ser seronegativos debido a que los anticuerpos no se han desarrollado. La situación inversa ocurre en los estadios tardíos de la infección donde los animales claramente tienen una infección bacteriológicamente negativa, pero serológicamente positiva. Así la serología tiene un alto potencial para la clasificación de los lotes, más que para un diagnóstico individual.

Estudios previos han mostrado que uno o más factores tales como el serotipo, tamaño del lote, estación y estado sanitario se encuentran asociados con la respuesta serológica y la duración de la infección por Salmonella en lotes de cerdos (Stege et al., 2000; Davies et al., 2004). Además, diferentes factores de riesgos se encuentran asociados a la presencia de Salmonella en cerdos, dentro de ellos la alimentación, la cual se ha relacionado en diferentes estudios. Así, se ha demostrado que el alimento húmedo desarrolla procesos de fermentación con la consecuente producción de ácido láctico encargado de la disminución de dichos factores de riesgo (Farzan et al., 2006).

Otro de los factores riesgo hace referencia al tamaño de las piaras, de acuerdo a lo descrito por Farzan et al., (2006) y Beloeil et al., (2007) en países como Francia y Dinamarca existe un incremento significativo del riesgo de seropositividad a Salmonella en granjas grandes si se comparan con las pequeñas piaras. El acceso a áreas exteriores puede incrementar el riesgo del contacto con suelo contaminado o animales de vida silvestre y por tanto el riesgo de infección con Salmonella (Jensen et al., 2004).

La seroprevalencia obtenida en granjas porcinas del Departamento del Tolima fue de $36.09 \%$, este valor es cercano a lo reportado en otros países. Así, Cardinale et al., (2010) demostraron una seroprevalencia de $40 \%$ en la isla Reunión. Igualmente, en un estudio de Grebeyes et al., (2008) en Carolina del Norte, Ohio y Wisconsin en Estados Unidos, se halló una seroprevalencia de 47\% (291/616) de Salmonella. En este mismo estudio se encontró una prevalencia altamente significativa de Salmonella en lotes de sistemas libres de antimicrobianos (54\%) comparados con los convencionales (39\%). En Dinamarca, Baptista et al., (2009) y Zheng et al., (2007) han descrito valores de seroprevalencia cercanos al 30\% para sistemas convencionales y con valores más bajos (rango de 0 - 39\%) para sistemas de pastoreo o mixtos. Por otra parte, dista de lo reportado por Rajic et al., (2007) quienes demostraron un $13.2 \%$ de seroprevalencia en granjas porcinas en Canadá y de lo descrito por Hautekiet et al., (2008) quienes reportan hasta un $68 \%$ de prevalencia de lote en Bélgica. En este último trabajo los autores modelan los factores de riesgo asociados a la seroprevalencia 
de Salmonella en las granjas, similar a lo descrito por Funk y Grebeyes (2004) y Gotter et al., (2012). En el departamento del Tolima, Henao y Ramírez (2010) demostraron que la falta de control de roedores y de control de animales de reemplazo constituían factores de riesgo para la presencia y seroprevalencia de Salmonella en granjas porcinas.

Estudios realizados por Davies et al., (1998) en diferentes ciclos de producción porcina describen una prevalencia de Salmonella entre el 18 y $22 \%$ en granjas de cerdos de cría y del $3.4 \%$ en granjas de cerdas de reemplazo. Sin embargo, esto difiere de lo hallado por Letellier et al., (1999) quienes reportaron una prevalencia del15.9\% en cerdas de reemplazo, indicándose así que el tipo de granja puede ser un factor importante en la prevalecía de animales positivos a Salmonella.

Vale la pena destacar que el hallazgo de animales seropositivos no implica que el animal tenga salmonelosis o que tenga Salmonella spp. en sangre, sino que existen anticuerpos anti-Salmonella spp. y no directamente el agente causal. Además, se denota que la salmonelosis comprende un cuadro clínico caracterizado por elevación de la temperatura corporal, inapetencia, diarrea mucosa profusa, entre otros (Hoelzer et al., 2011) y no la existencia per se de anticuerpos contra ella. De este modo, la prevalencia del $36.09 \%$ implica que los animales han estado expuestos a Salmonella spp. Recientemente o lo están al momento del muestreo, constituyéndose así un riesgo de contaminación de la carne y por ende un riesgo infección en la población, similar a lo descrito por De Busser et al., (2013).

Así, Siekkinen et al. (2006) demostraron que las bacterias patógenas que se diseminan en las plantas de sacrificio por contaminación cruzada pueden ser rastreadas al lote de cerdos original más que a la microflora de la planta de sacrificio.

Desde el 2001, en la Unión europea y en países como Dinamarca, incluyeron un promedio pesado de los resultados de seroprevalencia, variable denominada "Índice de Salmonella" (Salmonella index), utilizando suero y utilizando jugos cárnicos, calculada así que el resultado del mes más reciente fue dado tres veces más peso que los resultados de los dos meses previos. Además hubo cambios en los puntos de corte de la densidad óptica que incrementaron el número de animales reportados como seropositivos. Todos los lotes fueron asignados a 1 de 3 niveles cada mes. El nivel 1 incluía lotes que tenían un seroprevalencia a Salmonella "Aceptable, baja" (índice de Salmonella< 40), el nivel 2 correspondía a lotes con seroprevalencia (Moderada, aun aceptable) (Índice de Salmonella
$40>,<70)$ y el nivel 3 incluía lotes con proporción de seroprevalencia "inaceptable, alta" (Índice de Salmonella> 70) (Baptista et al., 2009). En producciones porcícolas de nivel 2 y 3 se establecieron medidas para reducir la prevalencia que van desde incluir la supervisión veterinaria constante así como multas. En Colombia, la vigilancia epidemiológica de la salmonelosis está empezando a ser normatizada, sin ser obligatoria, y se requiere evaluar estos índices con el fin de acceder a mercados internacionales así como fortalecer el conocimiento del comportamiento de la bacteria en los sistemas de producción.

Goldbach y Alban (2006) realizaron un análisis costo-beneficio de la implementación de medidas sanitarias, principalmente de higienización de las piaras en el control de Salmonella o salmonelosis en porcinos. Mediante modelos de simulación pudieron inferir que la desinfección con agua caliente puede ser una estrategia económicamente viable y sanitariamente relacionada con una disminución en la presencia de esta bacteria. Es de destacar que la viabilidad de estos estudios de costo-beneficio son fácilmente aplicable a países de la Unión Europea donde existe regulaciones y programas de vigilancia frente a esta bacteria en los cuales se estimula económicamente a sistemas de producción libres de ella. En Colombia, así como en otros países latinoamericanos este tipo de políticas están ausentes y se hace necesario que de manera integral las instituciones nacionales responsables fortalezcan los procesos de inspección, vigilancia y control que permitan minimizar los riesgos y asegurar que los alimentos sean inocuos y aptos para el consumo humano.

\section{Agradecimiento}

Al proyecto Código MADR: 040 2008W4497-3594: "Evaluación epidemiológica de la presencia de Salmonella spp. y determinación de factores de riesgo asociados a su presentación en granjas del Departamento del Tolima". Financiado por el Ministerio de Agricultura y Desarrollo Rural - Asociación Colombiana de Porcicultores.

\section{Referencias}

Alban L, Stege H, Dahl J. The new classification system for slaughter-pig herds in the Danish Salmonella Surveillance-and-Control Program. Prev vet med, 2002; 53: 133-146.

Alban-Baptista F, Møgelmose V, Sørensen L, Christensen H, Aabo S, Dahl J. Salmonella surveillance and control for finisher pigs and pork in Denmark - A case study. Food res int, 2012; 45: 656-665. 
Baptista FM, Alban L, Ersbøll AK, Nielsen LR. Factors affecting persistence of high Salmonella serology in Danish pig herds. Prev vet med, 2009; 92: 301-308

Barrow P, Methner U. 2013. Salmonella in Domestic Animals, 2da Edición. CABI. Oxfordshire. p. 82-83.

Beloeil P, Chauvin C, Proux K, Flavet C, Madec F, Alioum A. Risk Factors for Salmonella seroconversion of fattening pigs in farrow to finish herds. Vet res, 2007; 38: 835848.

Cardinale E, Maeder S, Porphyre V, Debin M. Salmonella in fattening pigs in Reunion Island: Herd prevalence and risk factors for infection. Prev vet med, 2010; 96: 281-285.

Cummings P, Sorvillo F, Kuo T. Salmonellosis related mortality in the Unites States, 1990-2006. Foodborne pathog Dis, 2010; 7(11):1393-1399.

Davies PR, Bovee FG, Funk JA, Morrow WE, Jones FT, Deen J. Isolation of Salmonella serotypes from feces of pigs raised in a multiple- site production system. J Am Vet Med Assoc, 1998; 212 (12): 1925-1929.

Davies RH, Dalziel R, Gibbens JC, Wilesmith JW, Ryan JMB, Evans SJ, Byrne C, Paiba GA, Pascoe SJS, Teale CJ. National survey for Salmonella in pigs, cattle and sheep at slaughter in Great Britain (1999-2000). J appl microbio, 2004; 96: 750-760.

De Busser E, De Zutter L, Dewulf J, Houf K, Dominiek M. Salmonella control in live pigs and slaughter. The Veterinary Journal, 2013; 196: 20-27.

Durango J, Arrieta G, Mattar S. Presencia de Salmonella spp. En un área del Caribe colombiano: un riesgo para la salud pública. Biomedica, 2004; 24: 89-96.

European Food Safety Authority (EFSA). Trends, Sources of Zoonoses, Zoonotic Agents, and Food-borne Outbreaks in the European Union in 2008. EFSA Journal, 2010; 8(1): 1496.

Farzan A, Friendship R, Dewey C, Warriner K, Poppe C, Klotins K. Prevalence of Salmonella spp. on Canadian pig farms using liquid or dry-feeding. Prev vet med, 2006; 73: 241-254.

Funk J, Gebreyes WA. Risk factors associated with Salmonella prevalence on swine farms. J swine health prod, 2004; 12(5): 246-251.

Funk JA, Harris IT, Davies PR. Comparison of fecal culture and Danish Mix-ELISA for determination of Salmonella enterica subsp. enterica prevalence in growing swine. Vet microbiol, 2005; 107: 115-126

Goldbach SG, Alban L. A cost-benefit analysis of Salmonella-control strategies in Danish pork production. Prev vet med, 2006; 77: 1-14

Gotter V, Kleina G, Koesters S, Kreienbrock L, Blaha T, Campe A. Main risk factors for Salmonella-infections in pigs in north-western Germany. Preventive Veterinary Medicine, 2012; [2012/03/16] Article in press. http:// dx.doi.org/10.1016/j.prevetmed.2012.03.016

Grebeyes WA, Bahnson PB, Funk JA, McKean J, Patchanee P. Seroprevalence of Trichinella, Toxoplasma, andSalmonella in antimicrobial-free and conventional swine pro- duction systems. Foodborne Pathog Dis, 2008; 5(2): 199-203.

Grimont P, Weill F. 2007. Antigenic formulae of the Salmonella serovars. Paris, Francia: WHO Collaborating Centre for Reference and Research on Salmonella, Institut Pasteur. Disponible en: http://www.pasteur.fr/ip/portal/ action/WebdriveActionEvent/oid/01s-000036-089 (acceso 23 de Mayo, 2014).

Guibourdenche $M$, Roggentin $P$, Mikoleit $M$, Fields $P$, Bockemu"hl J, Grimont P, Weill F, Supplement 20032007 (No. 47) to the White-Kauffmann-Le Minor scheme. Res microbiolo, 2010;161: 26-29.

Hautekiet V, Geert V, Marc V, Rony G. Development of a sanitary risk index for Salmonella seroprevalence in Belgian pig farms. Prev vet med, 2008; 86: 75-92.

Henao J, Ramírez E. 2010. Análisis de la aplicación de las buenas prácticas de producción (BPPs) en granjas porcícolas del departamento del Tolima. (Tesis Pregrado). Colombia. Universidad del Tolima.

Hendriksen RS, Vieira AR, Karlsmose S, Lo Fo Wong DM, Jensen $A B$, Wegener HC, Aarestrup FM. Global monitoring of Salmonella serovar distribution from the World Health Organization Global Foodborne Infections Network Country Data Bank: results of quality assured laboratories from 2001 to 2007. Foodborne Pathog Dis, 2011; 8: 887-900.

Hoelzer K, Moreno Al, Wiedmann M. Animal contact as a source of human non-typhoidal salmonellosis. Vet res, 2011; 42: 34-61.

Hollinger K. 2000. Epidemiology and Salmonellosis. En: Salmonella in domestic animals. Ed. Wray C, Wray C. CABI Publishing. New York. p. 341-353.

Hur J, Jawale C, Lee J. Antimicrobial resistance of Salmonella isolate from a food animals: a review. Food res int, 2012; 45: 819-830.

Jensen AN, Lodal J, Baggesen DL. High diversity of Salmonella serotypes found in an experiment with outdoor pigs. NJAS - Wageningen. Journal of Life Sciences, 2004; 52(2): 109-117

Kranker S, Alban L, Boes J, Dahl J. Longitudinal study of Salmonella enterica serotype Typhimurium infection in three Danish farrow-to-finish swine herds. J clin microbial, 2003; 41: 2282-2288.

Langridge GC, Wain J, Nair S. 2008. Invasive Salmonellosis in humans. Capítulo 8.6.2.2. En: Böck A, Curtiss R, Kaper JB, Neidhardt FC, Nyström T, et al., eds. EcoSalEscherichia coli and Salmonella: celular and molecular biology. Washington. ASM Press. Disponible en: http:// www.ecosal.org. (Acceso 23 de mayo, 2014).

Majowicz SE, Musto J, Scallan E, Angulo FJ, Kirk M, O'Brien SJ, Jones TF, Fazil A, Hoekstra RM. The global burden of nontyphidal Salmonella gastroenteritis. Clin infect dis, 2010; 50(6): 882-889.

Nowak B, von Müffling T, Chaunchom S, Hartung J. Salmonella contamination in pigs at slaughter and on the farm: A field study using an antibody ELISA test and a PCR technique. Int J Food Microbiol, 2007;115: 259-267. 
Quinn PJ, Markey BK, Carter ME, Donnelly WJ, Leonard FC. 2001. Veterinary Microbiology and Microbial Disease. Wiley-Blackwell. p. 113-118

Rajic A, Chow EY, Wu JT, Deckert AE, Reid-Smith R, Manninen K, Dewey CE, Fleury M, and McEwen SA. Salmonella infections in ninety Alberta swine finishing farms: serological prevalence, correlation between culture and serology, and risk factors for infection. Foodborne pathog dis, 2007; 4:169-177.

Rincon-Reyes O, Figueroa J. Prevalencia serológica de Salmonella enteritidis en la Población Canina del Municipio de Tunja, Colombia. Revista de Salud Pública, 2008; 10(3): 470-476.

Rusul G, Khair J, Radu S, Cheah CT, Yassin R. Prevalence of Salmonella in broilers at retail outlets, processing plants and farms in Malaysia. Int J Food Microbiol, 1996; 33:183-194

Sallam KL, Mohammed MA, Hassan MA, Tamura T. Prevalence, molecular identification and antimicrobial resistance profile of Salmonella serovars isolate from retail beef products in Mansura, Egypt. Food control, 2014; 38: $209-214$

Siekkinen KM, Nuotio L, Ranta J, Laukkanen R, Hellström S, Korkeala H, Maijala R. Assessing hygiene proficiency on organic and conventional pig farms regarding pork safety: a pilot study in Finland. Livest sci, 2006; 104: 193-202.

Sørensen LL, Alban L, Nielsen B, Dahl J. The correlation between Salmonella serology and isolation of Salmonella in Danish pigs at slaughter. Veterinary Microbiology, 2004; 101:131-141.

Stege $\mathrm{H}$, Christensen J, Baggesen DL, Enué C, Nielsen JP, Willeberg P. Prevalence of subclinical Salmonella enterica infection in Danish finishing pig herds. Prev vet med, 2000; 44: 175-188.

Thrusfield M. 2007. Veterinary epidemiology. $3^{\text {rd }}$ ed. Blackwell Science, Oxford, UK, p.182-184.

Tunes MJ, Blas G. 2007. Salmonella. En: Microbiología Veterinaria. Ed. Stanchi O. Intermedica Editorial. Buenos Aires, Argentina. p. 210-214.

Wong LF, Dahl J, van der Wolf PJ, Wingstrand A, Leontides L, von Altrock A. Recovery of Salmonella enterica from seropositive finishing pig herds. Vet microbial, 2003; 97: 201-214.

Zheng DM, Bonde M, Sørensen JT. Association between the proportion of Salmonella slaughter pigs and the presence of herd level risk factors for introduction and transmission of Salmonella in 34 Danish organic, outdoor (non-organic) and indoor finishing-pig farms. Livest Sci, 2007;106: 189-199. 